\title{
Stability of Einstein static universe in gravity theory with a non-minimal derivative coupling
}

\author{
Qihong Huang ${ }^{1,2}$, Puxun $\mathbf{W u}^{1,3}$, Hongwei Yu${ }^{1, a}$ \\ ${ }^{1}$ Department of Physics and Synergetic Innovation Center for Quantum Effects and Applications, Hunan Normal University, Changsha, \\ Hunan 410081, China \\ ${ }^{2}$ School of Physics and Electronic Science, Zunyi Normal College, Zunyi 563006, China \\ ${ }^{3}$ Center for High Energy Physics, Peking University, Beijing 100080, China
}

Received: 30 June 2017 / Accepted: 7 January 2018 / Published online: 20 January 2018

(C) The Author(s) 2018. This article is an open access publication

\begin{abstract}
The emergent mechanism provides a possible way to resolve the big-bang singularity problem by assuming that our universe originates from the Einstein static (ES) state. Thus, the existence of a stable ES solution becomes a very crucial prerequisite for the emergent scenario. In this paper, we study the stability of an ES universe in gravity theory with a non-minimal coupling between the kinetic term of a scalar field and the Einstein tensor. We find that the ES solution is stable under both scalar and tensor perturbations when the model parameters satisfy certain conditions, which indicates that the big-bang singularity can be avoided successfully by the emergent mechanism in the non-minimally kinetic coupled gravity.
\end{abstract}

\section{Introduction}

Although the standard cosmological model achieves great success, it still suffers from several theoretical problems. The attempt to resolve theses problems lead to the invention of the theory of inflation [1-3], which settles successfully most of the problems in the standard cosmological model, but leaves the big-bang singularity problem open. To avoid this problem, some theories, such as the pre-big bang [4,5], the cyclic scenario [6-9] and the emergent scenario $[10,11]$, have been proposed. The emergent scenario, proposed by Ellis et al., in the framework of general relativity $[10,11]$, assumes that the universe originates from an Einstein static (ES) state rather than a big bang singularity. Therefore, it requires that the universe can stay in an ES state past eternally, and exit this static state naturally and then evolve to a subsequent inflationary era. Apparently, a very crucial prerequisite for the emergent

a e-mail: hwyu@hunnu.edu.cn scenario is the existence of a stable ES solution under various perturbations, such as quantum fluctuations. However, in the framework of general relativity, the emergent mechanism is not as successful as one expected in avoiding the big-bang singularity since there is no stable ES solution in a Friedmann universe with a scalar field minimally coupled with gravity [12-15].

A natural generalization of the minimally coupled gravity is to assume a non-minimal coupling between the scalar field and the curvature, which can be generated naturally when quantum corrections are considered and is essential for the renormalizability of the scalar-field theory in curved space. This non-minimally coupled scalar field has been suggested to be responsible for both the early cosmic inflation $[16,17]$ and the present accelerated expansion [18-20]. If the coupling is a general function of the scalar field, the resulting theory is called the scalar-tensor theory [21-23]. The popular modified gravity, $f(R)$ gravity [24,25], can be cast into a special form of the Brans-Dicke theory, which is a particular example of the scalar-tensor theory [26,27], with a potential for the effective scalar-field degree of freedom. Let us also note that a pioneering inflation model was constructed in a $f(R)$ theory by Starobinsky [1], which allows a graceful exit from inflation to the subsequent radiation dominated stage and produces a very good fit to existing CMB observational data [28].

It has been found that in $f(R)$ theory the inhomogeneous scalar perturbations break the stability of the ES solution which is stable under homogeneous scalar perturbations [2934]. Recently, Miao et al. [35] found that there is no stable ES solution when scalar perturbations and tensor ones are considered together in the scalar-tensor theory of gravity with a normal perfect fluid, such as radiation or pressureless matter. It is worthy to note that the stability of ES solutions have also been analyzed in some other theories [36-82]. 
Except for the coupling between the scalar field and the curvature, there are many other coupling, such as the coupling between the kinetic term of the scalar field and the Einstein tensor. This non-minimal derivative coupling has been discussed extensively in cosmology. For example, it can provide an inflationary mechanism [83-93], explain both a quaside Sitter phase and an exit from it without any fine-tuned potential [94], and behave as a dark matter [95,96] or a dark energy [97-100]. Recently, the stability of ES solutions in the non-minimally derivative coupled gravity have been studied in [67]. However, in [67] only a very special case of $\dot{\phi}=0$ is considered, which is not a general result derived from the conditions of static state solution, where $\dot{\phi}=\mathrm{d} \phi / \mathrm{d} t$ with $\phi$ and $t$ being the scalar field and the cosmic time, respectively. In addition, only the homogeneous scalar perturbations and tensor perturbations are considered in [67]. Thus, for the nonminimally kinetic coupled gravity, it is unclear whether the ES solution remain to be stable against inhomogeneous scalar perturbations and what the effect the special condition $\dot{\phi}=0$ has on the stable regions, and this motivates us to the present work.

The paper is organized as follows. In Sect. 2, we give the field equations of gravity theory with a non-minimal derivative coupling and the ES solution. In Sect. 3, we analyze the stability of ES solution under tensor perturbations. In Sect. 4, the homogeneous and inhomogeneous scalar perturbations are considered. Finally, our main conclusions are presented in Sect. 5. Throughout this paper, unless specified, we adopt the metric signature $(-,+,+,+)$. Latin indices run from 0 to 3 and the Einstein convention is assumed for repeated indices.

\section{The field equations and Einstein static solution}

The action of the non-minimally derivative coupled gravity has the form [83,101-103]

$$
\begin{aligned}
S= & \int \mathrm{d}^{4} x \sqrt{-g}\left[\frac{R}{8 \pi G}-\left(g_{\mu \nu}+\kappa G_{\mu \nu}\right)\right. \\
& \left.\nabla^{\mu} \phi \nabla^{v} \phi-2 V(\phi)\right]+S_{m},
\end{aligned}
$$

where $R$ is the Ricci curvature scalar, $G$ is the Newtonian gravitational constant, $g_{\mu \nu}$ is the metric tensor with $g$ being its trace, $G_{\mu \nu}$ is the Einstein tensor, $V(\phi)$ is the potential of the scalar field $\phi, \kappa$ stands for the coupling parameter with dimension of (length) ${ }^{2}$, and $S_{m}$ represents the action of a perfect fluid.

Varying the action (1) with respect to the metric tensor $g_{\mu \nu}$ and the scalar field $\phi$, respectively, one can obtain two independent equations:

$G_{\mu \nu}=8 \pi G\left[T_{\mu \nu}^{(m)}+T_{\mu \nu}^{(\phi)}+\kappa \vartheta_{\mu \nu}\right]$ and

$\left(g^{\mu \nu}+\kappa G^{\mu \nu}\right) \nabla_{\mu} \nabla_{\nu} \phi=V_{, \phi}$,

where $V_{, \phi}=\frac{\mathrm{d} V}{\mathrm{~d} \phi}, T_{\mu \nu}^{(m)}$ is the energy-momentum tensor of the perfect fluid, and

$$
\begin{aligned}
T_{\mu \nu}^{(\phi)}= & \nabla_{\mu} \phi \nabla_{\nu} \phi-\frac{1}{2} g_{\mu \nu}(\nabla \phi)^{2}-g_{\mu \nu} V \\
\vartheta_{\mu \nu}= & -\frac{1}{2} \nabla_{\mu} \phi \nabla_{\nu} \phi R+2 \nabla_{\alpha} \phi \nabla_{(\mu} \phi R_{\nu)}^{\alpha}+\nabla^{\alpha} \phi \nabla^{\beta} \phi R_{\mu \alpha \nu \beta} \\
& +\nabla_{\mu} \nabla^{\alpha} \phi \nabla_{\nu} \nabla_{\mu} \phi-\nabla_{\mu} \nabla_{\nu} \phi \square \phi-\frac{1}{2}(\nabla \phi)^{2} G_{\mu \nu} \\
& +g_{\mu \nu}\left[-\frac{1}{2} \nabla^{\alpha} \nabla^{\beta} \phi \nabla_{\alpha} \nabla_{\beta} \phi+\frac{1}{2}(\square \phi)^{2}\right. \\
& \left.-\nabla_{\alpha} \phi \nabla_{\beta} \phi R^{\alpha \beta}\right] .
\end{aligned}
$$

Here, $(\nabla \phi)^{2}=\nabla_{\alpha} \phi \nabla^{\alpha} \phi$ and $\square \phi=\nabla_{\alpha} \nabla^{\alpha} \phi$.

To find an ES solution, we consider a homogeneous and isotropic universe described by the Friedmann-LemaîtreRobertson-Walker metric,

$\mathrm{d} s^{2}=a(\eta)^{2}\left[-\mathrm{d} \eta^{2}+\gamma_{i j} \mathrm{~d} x^{i} \mathrm{~d} x^{j}\right]$

where $\eta$ is the conformal time, $a(\eta)$ denotes the conformal scale factor, and $\gamma_{i j}$ represents the metric on the three-sphere,

$\gamma_{i j} \mathrm{~d} x^{i} \mathrm{~d} x^{j}=\frac{\mathrm{d} r^{2}}{1-K r^{2}}+r^{2}\left(\mathrm{~d} \theta^{2}+\sin ^{2} \theta \mathrm{d} \phi^{2}\right)$.

Here $K=+1,0,-1$ corresponds to a closed, flat, and open universe, respectively. The $(00)$ and $(i j)$ components of Eq. (2) give

$$
\begin{aligned}
\mathcal{H}^{2}+K= & \frac{8 \pi G}{3} a^{2}\left[\rho+\frac{1}{2 a^{2}} \phi^{\prime 2}+V\right. \\
& \left.-\kappa \frac{3}{2 a^{4}}\left(3 \mathcal{H}^{2}+K\right) \phi^{\prime 2}\right], \\
2 \mathcal{H}^{\prime}+\mathcal{H}^{2}+K= & -8 \pi G a^{2}\left[p+\frac{1}{2 a^{2}} \phi^{\prime 2}-V+\kappa \frac{1}{2 a^{4}}\right. \\
& \left.\times\left(2 \mathcal{H}^{\prime}-3 \mathcal{H}^{2}-K+4 \mathcal{H} \frac{\phi^{\prime \prime}}{\phi^{\prime}}\right) \phi^{\prime 2}\right],
\end{aligned}
$$

where $\rho$ and $p$ are the energy density and the pressure of the perfect fluid, respectively, $p=w \rho$ with $w$ being a constant, $\mathcal{H}=\frac{1}{a} \frac{\mathrm{d} a}{\mathrm{~d} \eta}$ and ' denotes the derivative with respect to the conformal time $\eta$. From Eq. (3) we obtain the dynamical equation of the scalar field:

$$
\frac{1}{a^{2}}\left(\phi^{\prime \prime}+2 \mathcal{H} \phi^{\prime}\right)-\kappa \frac{3}{a^{4}}\left[\left(\mathcal{H}^{2}+K\right) \phi^{\prime \prime}+2 \mathcal{H} \mathcal{H}^{\prime} \phi^{\prime}\right]=-V_{, \phi}
$$




\subsection{Einstein static solution}

The ES solution requires that the conditions of $a=a_{0}=$ constant and $a_{0}^{\prime}=a_{0}^{\prime \prime}=0$ should be satisfied. Then Eq. (8) can be reduced to

$\frac{K}{a_{0}^{2}}=\frac{8 \pi G}{3}\left(\rho_{0}+\frac{1}{2 a_{0}^{2}} \phi_{0}^{\prime 2}+V_{0}-\frac{3}{2 a_{0}^{4}} \kappa K \phi_{0}^{\prime 2}\right)$,

where the subscript 0 represents the value at the ES state. It is easy to see that to obtain an ES state $\rho_{0}, V_{0}$ and $\phi_{0}^{\prime 2}$ must be constant. From Eqs. (9) and (10) we have

$$
\begin{aligned}
& \frac{K}{a_{0}^{2}}=-8 \pi G\left(p_{0}+\frac{1}{2 a_{0}^{2}} \phi_{0}^{\prime 2}-V_{0}-\frac{1}{2 a_{0}^{4}} \kappa K \phi_{0}^{\prime 2}\right), \\
& \left.\frac{\mathrm{d} V}{\mathrm{~d} \phi}\right|_{\phi=\phi_{0}}=0 .
\end{aligned}
$$

Thus, the scalar field with a constant speed moves on a constant potential in the ES state. However, in [67], a special case $\phi_{0}^{\prime}=0$ was considered and $\phi_{0}=0$ was assumed. Combining Eqs. (11) and (12) leads to

$\frac{K}{a_{0}^{2}}=4 \pi G \rho_{0}(1+w)+4 \pi G \phi_{0}^{\prime 2} \frac{1}{a_{0}^{2}}-8 \pi G \phi_{0}^{\prime 2} \kappa \frac{K}{a_{0}^{4}}$,

which indicates that $K \neq 0$ for the existence of an ES solution since $\frac{1}{a^{2}} \phi^{\prime 2}=\left(\frac{\mathrm{d} \phi}{\mathrm{d} t}\right)^{2}$. After introducing two new constants

$F=4 \pi G \phi_{0}^{\prime 2} \frac{1}{a_{0}^{2}}=4 \pi G \dot{\phi}_{0}^{2}, \quad \rho_{s}=4 \pi G \rho_{0}$.

Equation (14) can be re-expressed as

$\frac{1}{a_{0}^{2}}=\frac{F+(1+w) \rho_{s}}{(1+2 \kappa F) K}$.

Since $a_{0}$ and $\rho_{0}$ should take positive values, the existence conditions of ES solutions are $a_{0}^{2}>0$ and $\rho_{s}>0$. For the case $\kappa<0$, when $K=1$, we find that the existence of ES solutions requires

$$
\begin{aligned}
& w<-1, \quad 0<F<-\frac{1}{2 \kappa}, \quad 0<\rho_{s}<-\frac{F}{1+w}, \\
& w<-1, \quad F>-\frac{1}{2 \kappa}, \quad \rho_{s}>-\frac{F}{1+w}, \\
& w=-1, \quad 0<F<-\frac{1}{2 \kappa}, \quad \rho_{s}>0, \\
& \text { or } \quad w>-1, \quad 0 \leq F<-\frac{1}{2 \kappa}, \quad \rho_{s}>0 .
\end{aligned}
$$

For $K=-1$, the conditions become

$$
\begin{aligned}
& w<-1, \quad 0 \leq F<-\frac{1}{2 \kappa}, \quad \rho_{s}>-\frac{F}{1+w}, \\
& w<-1, \quad F>-\frac{1}{2 \kappa}, \quad 0<\rho_{s}<-\frac{F}{1+w}, \\
& \text { or } \quad w \geq-1, \quad F>-\frac{1}{2 \kappa}, \quad \rho_{s}>0 .
\end{aligned}
$$

For the case of $\kappa>0, a_{0}^{2}>0$ and $\rho_{s}>0$ lead to

$$
\begin{aligned}
& w<-1, \quad F>0, \quad 0<\rho_{s}<-\frac{F}{1+w}, \\
& w=-1, \quad F>0, \quad \rho_{s}>0, \\
& \text { or } \quad w>-1, \quad F \geq 0, \quad \rho_{s}>0,
\end{aligned}
$$

when $K=1$, and

$w<-1, \quad F \geq 0, \quad \rho_{s}>-\frac{F}{1+w}$,

when $K=-1$.

In the following we will discuss the stability of ES solutions under the scalar and tensor perturbations. The tensor perturbations will be analyzed firstly since they are relatively easy to handle.

\section{Tensor perturbations}

For the tensor perturbations, the perturbed metric has the following form [104]:

$\mathrm{d} s^{2}=a(\eta)^{2}\left[-\mathrm{d} \eta^{2}+\left(\gamma_{i j}+2 h_{i j}\right) \mathrm{d} x^{i} \mathrm{~d} x^{j}\right]$.

For convenience, we perform a harmonic decomposition for the perturbed variable $h_{i j}$,

$h_{i j}=H_{T, n l m}(\eta) Y_{i j, n l m}\left(\theta^{k}\right)$,

where summations over $n, m, l$ are implied. The quantum numbers $m$ and $l$ will be suppressed hereafter as they do not enter the differential equation for the perturbations. The harmonic function $Y_{n}=Y_{n l m}\left(\theta^{i}\right)$ satisfies [105]

$$
\begin{aligned}
\Delta Y_{n} & =-k^{2} Y_{n} \\
& =\left\{\begin{array}{lll}
-n(n+2) Y_{n}, & n=0,1,2, \ldots, & K=+1, \\
-n^{2} Y_{n}, & n^{2} \geq 0, & K=0, \\
-\left(n^{2}+1\right) Y_{n}, & n^{2} \geq 0, & K=-1 .
\end{array}\right.
\end{aligned}
$$

Here, $\Delta$ represents the 3-dimensional spatial Laplacian operator. The spectrum of the perturbation modes is discrete for $K=1$, while it is continuous for $K=0$ or -1 .

Substituting the metric given in Eq. (21) into the field equations (Eq. (2)) leads to

$$
\begin{aligned}
& \left(1+4 \pi G \phi^{\prime 2} \frac{\kappa}{a^{2}}\right) H_{T}^{\prime \prime}+\left(2 \mathcal{H}+8 \pi G \phi^{\prime} \phi^{\prime \prime} \frac{\kappa}{a^{2}}\right) H_{T}^{\prime} \\
& +\left(1-4 \pi G \phi^{\prime 2} \frac{\kappa}{a^{2}}\right)\left(k^{2}+2 K\right) H_{T}=0 .
\end{aligned}
$$

Under the ES background, this equation can be simplified as

$H_{T}^{\prime \prime}+B H_{T}=0, \quad B \equiv \frac{(1-\kappa F)}{(1+\kappa F)}\left(k^{2}+2 K\right)$.

To obtain the stable ES solution against tensor perturbations, $B>0$ must be satisfied for any $k$. For the case of $\kappa<0$, 
when $K=1$, we find that the restriction condition $B>0$ gives

$k^{2} \geq 0, \quad 0 \leq F<-\frac{1}{\kappa}$.

While, when $K=-1$, the stable ES solution requires

$$
\begin{aligned}
& k^{2}>2, \quad 0 \leq F<-\frac{1}{\kappa}, \\
& \text { or } 1 \leq k^{2}<2, \quad F>-\frac{1}{\kappa} .
\end{aligned}
$$

For the case of $\kappa>0, B>0$ leads to

$k^{2} \geq 0, \quad 0 \leq F<\frac{1}{\kappa}$,

when $K=1$, and

$k^{2}>2, \quad 0 \leq F<\frac{1}{\kappa}$,

or $1 \leq k^{2}<2, \quad F>\frac{1}{\kappa}$,

when $K=-1$. It is easy to see that the stable ES solution exists only in the case of spatially closed universe $(K=$ 1). Thus, in the following analysis $K=1$ is considered. Combining the existence conditions given in Eqs. (17) and (19) and the stability conditions under tensor perturbations, we see that $w, F$ and $\rho_{s}$ should satisfy

$w<-1, \quad 0<F<-\frac{1}{2 \kappa}, \quad 0<\rho_{s}<-\frac{F}{1+w}$,

$w<-1, \quad-\frac{1}{2 \kappa}<F<-\frac{1}{\kappa}, \quad \rho_{s}>-\frac{F}{1+w}$,

$w=-1, \quad 0<F<-\frac{1}{2 \kappa}, \quad \rho_{s}>0$,

or $w>-1, \quad 0 \leq F<-\frac{1}{2 \kappa}, \quad \rho_{s}>0$,

for $\kappa<0$, and

$w<-1, \quad 0<F<\frac{1}{\kappa}, \quad 0<\rho_{s}<-\frac{F}{1+w}$,

$w=-1, \quad 0<F<\frac{1}{\kappa}, \quad \rho_{s}>0$,

or $w>-1, \quad 0 \leq F<\frac{1}{\kappa}, \quad \rho_{s}>0$,

for $\kappa>0$.

\section{Scalar perturbations}

To analyze the stability of ES solutions under scalar perturbations, we consider the perturbed metric:

$\mathrm{d} s^{2}=a(\eta)^{2}\left[-(1+2 \Psi) \mathrm{d} \eta^{2}+(1+2 \Phi) \gamma_{i j} \mathrm{~d} x^{i} \mathrm{~d} x^{j}\right]$, where the Newton gauge has been used, $\Psi$ is the Bardeen potential and $\Phi$ denotes the perturbation to the spatial curvature.

Using the above perturbed metric and the field equations given in Eqs. (2) and (3), we obtain the following perturbation equations:

$$
\begin{aligned}
& \frac{1}{4 \pi G a_{0}^{2}}\left(\nabla^{2} \Phi+3 \Phi\right) \\
& =-\delta \rho+\frac{1}{a_{0}^{2}}\left(\phi_{0}^{\prime 2} \Psi-\phi_{0}^{\prime} \delta \phi^{\prime}\right) \\
& \quad+\kappa \frac{1}{a_{0}^{4}}\left[3 \phi_{0}^{\prime} \delta \phi^{\prime}-\phi_{0}^{\prime 2} \nabla^{2} \Phi-3(\Psi+\Phi) \phi_{0}^{\prime 2}\right], \\
& -(\Psi+\Phi)=4 \pi G \phi_{0}^{\prime 2} \kappa \frac{1}{a_{0}^{2}}(\Psi-\Phi), \\
& \frac{3}{a_{0}^{2}}\left(-\Phi^{\prime \prime}+\Phi\right)+\frac{1}{a_{0}^{2}} \nabla^{2}(\Psi+\Phi) \\
& =4 \pi G\left\{3 \delta p-\frac{3}{a_{0}^{2}}\left(\phi_{0}^{\prime 2} \Psi-\phi_{0}^{\prime} \delta \phi^{\prime}\right)\right. \\
& \quad+\kappa \frac{1}{a_{0}^{4}}\left[\phi_{0}^{\prime 2} \nabla^{2}(\Phi-\Psi)+3 \phi_{0}^{\prime 2} \Phi^{\prime \prime}\right. \\
& \left.\left.\quad+3(\Psi+\Phi) \phi_{0}^{\prime 2}-3 \phi_{0}^{\prime} \delta \phi^{\prime}\right]\right\} \\
& \quad\left(1-\kappa \frac{3}{a_{0}^{2}}\right) \delta \phi^{\prime \prime}-\left(1-\kappa \frac{1}{a_{0}^{2}}\right) \nabla^{2} \delta \phi \\
& \quad-\left[\left(\Psi^{\prime}-3 \Phi^{\prime}\right)-\kappa \frac{3}{a_{0}^{2}}\left(\Psi^{\prime}-\Phi^{\prime}\right)\right] \phi_{0}^{\prime}=0 .
\end{aligned}
$$

Here, the perturbation of the scalar field $\phi \rightarrow \phi_{0}+\delta \phi$ is considered. For the perfect fluid, the perturbation of its energy-momentum tensor can be expressed as

$\delta T_{\nu}^{\mu(m)}=\delta \rho u^{\mu} u^{\nu}+u^{\mu} D_{\nu} q+u_{\nu} D^{\mu} q+\delta p P_{\nu}^{\mu}$,

where $u^{\mu}$ is the four-velocity of matter and $q$ is related to the perturbation of the spatial component of this four-velocity. The projection tensor $P_{\nu}^{\mu}$ and the derivative $D_{\mu}$ are defined as

$P_{v}^{\mu}=\delta_{v}^{\mu}+u_{v}^{\mu}$,

$D_{\mu}=P_{\mu}^{\alpha} \partial_{\alpha}=\partial_{\mu}+u_{\mu} u^{\alpha} \partial_{\alpha}$.

The relation between the density and pressure perturbations is

$\delta p=c_{s}^{2} \rho_{0} \delta$,

where $\delta=\delta \rho / \rho_{0}$ and $c_{s}^{2}=w$ is the sound speed.

Similar to the case of tensor perturbations, we perform a harmonic decomposition for the perturbed variables 
$\Psi=\Psi_{n}(\eta) Y_{n}\left(\theta^{i}\right), \quad \Phi=\Phi_{n}(\eta) Y_{n}\left(\theta^{i}\right)$,

$q=q_{n}(\eta) Y_{n}\left(\theta^{i}\right)$,

$\delta=\delta_{n}(\eta) Y_{n}\left(\theta^{i}\right), \quad \delta \phi=\delta \phi_{n}(\eta) Y_{n}\left(\theta^{i}\right)$.

Combining Eqs. (33), (34), (35) and (36) gives two independent perturbed equations,

$\Phi_{n}^{\prime \prime}+b_{11} \Phi_{n}+a_{12} \delta \phi_{n}^{\prime}=0$,

$\delta \phi_{n}^{\prime \prime}+b_{22} \delta \phi_{n}+a_{21} \Phi_{n}^{\prime}=0$,

with

$$
\begin{aligned}
b_{11}= & w k^{2}-\frac{\kappa F(1-\kappa F)(w-1)}{\frac{\kappa}{a_{0}^{2}}(1+\kappa F)^{2}} \\
& -\frac{2 \kappa^{2} F^{2}(3 w-1)+(1+\kappa F)(3 w+1)}{(1+\kappa F)^{2}}, \\
a_{12}= & -\frac{F\left[(1-3 w) \frac{\kappa}{a_{0}^{2}}+(w-1)\right]}{\frac{1}{a_{0}^{2}}(1+\kappa F)} \frac{1}{\phi_{0}^{\prime}}, \\
b_{22}= & \frac{1-\frac{\kappa}{a_{0}^{2}} k^{2},}{1-3 \frac{\kappa}{a_{0}^{2}}} \\
a_{21}= & 2\left(\frac{1}{1-3 \frac{\kappa}{a_{0}^{2}}}+\frac{1}{1+\kappa F}\right) \phi_{0}^{\prime} .
\end{aligned}
$$

Introducing two new variables $\delta \varphi=\delta \phi^{\prime}$ and $\Upsilon=\Phi^{\prime}$, Eqs. (42) and (43) can be rewritten as
Since $b_{22}=0$ and $M^{2}=N$ when $k^{2}=0$, the homogeneous scalar perturbations require

$\mu^{2}=\frac{-M-\sqrt{N}}{2}=-M=-b_{11}+a_{12} a_{21}<0$.

\subsection{Stability}

For the scalar perturbations, the analysis of the stability of ES solutions is very complicated. To simplify discussions, we will consider the constraints from the tensor perturbations and the existence conditions obtained in the previous sections, in which it is found that the ES solution is stable under the conditions of $K=1$ and Eqs. (30) and (31).

\subsection{1 $\kappa<0$}

From Eq. (49), we see that the stability conditions under homogeneous scalar perturbations are

$$
\begin{aligned}
& F=0, \quad-1<w<-\frac{1}{3}, \quad \rho_{s}>0, \\
& \text { or } 0<F<-\frac{1}{4 \kappa}, \quad-1<w<\frac{-1+2 \kappa F}{3+6 \kappa F}, \\
& \rho_{s}>\lambda_{+},
\end{aligned}
$$

where

$$
\begin{aligned}
\lambda_{ \pm}= & \frac{1+3 w+\kappa F[-11+3 w-2 \kappa F(1+3 w)]}{6 \kappa(1+w)[1+3 w+\kappa F(-2+6 w)]} \\
& \pm \frac{1}{6} \sqrt{\frac{(1+2 \kappa F)^{2}\left[1+(22-23 \kappa F) \kappa F+6 w+6 \kappa F(10+13 \kappa F) w+9(-1+\kappa F)^{2} w^{2}\right]}{\kappa^{2}(1+w)^{2}[1+3 w+\kappa F(-2+6 w)]^{2}}}
\end{aligned}
$$

$$
\begin{aligned}
& \Phi_{n}^{\prime}-\Upsilon_{n}=0, \\
& \Upsilon_{n}^{\prime}+b_{11} \Phi_{n}+a_{12} \delta \varphi_{n}=0, \\
& \delta \phi_{n}^{\prime}-\delta \varphi_{n}=0, \\
& \delta \varphi_{n}^{\prime}+b_{22} \delta \phi_{n}+a_{21} \Upsilon_{n}=0 .
\end{aligned}
$$

The stability of ES solutions is determined by the eigenvalues of the coefficient matrix, which is

$\mu^{2}=\frac{-M \pm \sqrt{N}}{2}$

where

$M=b_{11}+b_{22}-a_{12} a_{21}$,

$N=-4 b_{11} b_{22}+\left(b_{11}+b_{22}-a_{12} a_{21}\right)^{2}$.

If $\mu^{2}<0$, a small perturbation from the ES state will result in an oscillation around this state rather than an exponential deviation. Thus, the corresponding ES solution is stable. Otherwise, it is unstable. $\mu^{2}<0$ gives the stability conditions under scalar perturbations

$M>0, \quad N>0, \quad M^{2}>N$.
For $0<F<-\frac{1}{4 \kappa}$, one can obtain $-1<\frac{-1+2 \kappa F}{3+6 \kappa F}<-\frac{1}{3}$, which means that $w$ is negative.

For the inhomogeneous scalar perturbations, the physical modes have $n \geq 2$, which gives $k^{2} \geq 8$ since the $n=1$ mode corresponds to a gauge degree of freedom related to a global rotation. For $F=0$ and $k^{2}=8$, we see that the regions of $w$ and $\rho_{s}$ are

$$
\begin{aligned}
& \frac{1}{5}<w \leq \frac{11}{15}, \quad \rho_{s}>0, \\
& \frac{11}{15}<w<\frac{9}{5}, \quad 0<\rho_{s}<\frac{-9+5 w}{-11 \kappa+4 \kappa w+15 \kappa w^{2}}, \\
& \frac{11}{15}<w<\frac{9}{5}, \quad \rho_{s}>\frac{-9+5 w}{-11 \kappa+4 \kappa w+15 \kappa w^{2}}, \\
& \text { or } \quad w \geq \frac{9}{5}, \quad \rho_{s}>0 .
\end{aligned}
$$

When $0<F<-\frac{1}{4 \kappa}$ and $k^{2}=8$, we find that $w$ and $\rho_{s}$ need to satisfy 
$0<w<\frac{1+\kappa F-2 \kappa^{2} F^{2}}{5+13 \kappa F+2 \kappa^{2} F^{2}}$,

$0<\rho_{s}<\frac{-4 F w\left[1+3 \kappa F+\kappa^{2} F^{2}\right]}{(1+w)\left[-1+5 w+\kappa F(-1+13 w)+2 \kappa^{2} F^{2}(1+w)\right]}$,

or $\quad w \geq \frac{1+\kappa F-2 \kappa^{2} F^{2}}{5+13 \kappa F+2 \kappa^{2} F^{2}}>0, \quad \rho_{s}>0$.

Obviously, a positive $w$ is required for the stable ES solution under the inhomogeneous scalar perturbations, which conflicts with the conditions given by the homogeneous scalar perturbations and the tensor ones. Thus, there is no stable ES solution in the case of $\kappa<0$.

\section{$4.1 .2 \kappa>0$}

When $\kappa>0$, the results are summarized in Table 1 where the conditions shown in Eq. (31) have been considered together. The constants $\xi$ and $\zeta$ are defined as

$$
\begin{aligned}
& \xi=\frac{-11-30 w+9 w^{2}}{\kappa\left(-23+78 w+9 w^{2}\right)}+12 \sqrt{\frac{1+5 w+3 w^{2}-9 w^{3}}{\kappa^{2}\left(-23+78 w+9 w^{2}\right)^{2}}}, \\
& \zeta=\frac{4 F\left(1+\kappa F+\kappa^{2} F^{2}\right)}{(1+w)[-1-3 w+\kappa F(11-3 w+2 \kappa F+6 \kappa F w)]}
\end{aligned}
$$

Now we consider the contribution from inhomogeneous scalar perturbations. We find that there is no stable ES solution for $w \leq 0$ since $M>0$ and $M^{2}-N>0$ cannot be satisfied simultaneously when $k^{2}=8$. Since the expressions are complicated, we do not show them here.

When $0<w<\frac{1}{9}$, from Table 1 one can see that there are four different kinds of stability conditions under homogeneous scalar perturbations. We will analyze inhomogeneous scalar perturbations under these conditions, respectively.

(i) $0<F<\frac{1+3 w}{2 \kappa-6 \kappa w}$ and $\frac{1-\kappa F}{3 \kappa+3 \kappa w}<\rho_{s}<\lambda_{+}$. The stability condition $M>0$ under inhomogeneous scalar perturbations requires $\rho_{s}$ to satisfy

$$
0<\rho_{s}<\frac{1-\kappa F}{3 \kappa+3 \kappa w}, \quad \text { or } \rho_{s}>\varpi
$$

where
Table 1 Summary of the combinations of the stability conditions under homogeneous scalar perturbations and that given in Eq. (31) with $K=1$ and $\kappa>0$

\begin{tabular}{lll}
\hline$w$ & $F$ & $\rho_{s}$ \\
\hline$-1<w<-\frac{1}{3}$ & $F=0$ & $\rho_{s}>0$ \\
& $0<F<\xi$ & $\lambda_{-}<\rho_{s}<\lambda_{+}$ \\
& $0<F<\frac{1}{\kappa}$ & $\rho_{s}>\frac{1-\kappa F}{3 \kappa+3 \kappa w}$ \\
$w=-\frac{1}{3}$ & $0<F<\frac{1}{\kappa}$ & $\rho_{s}>\frac{1-\kappa F}{2 \kappa}$ \\
$-\frac{1}{3}<w<0$ & $0<F<\frac{1+3 w}{2 \kappa-6 \kappa w}$ & $\frac{1-\kappa F}{3 \kappa+3 \kappa w}<\rho_{s}<\lambda_{+}$ \\
& $F=\frac{1+3 \kappa w}{2 \kappa-6 \kappa w}$ & $\frac{1-\kappa F}{3 \kappa+3 \kappa w}<\rho_{s}<\zeta$ \\
& $\frac{1+3 \kappa w}{2 \kappa-6 \kappa w}<F \leq \xi$ & $\frac{1-\kappa F}{3 \kappa+3 \kappa w}<\rho_{s}<\lambda_{-}$ \\
& $\frac{1+3 w}{2 \kappa-6 \kappa w}<F<\xi$ & $\rho_{s}>\lambda+$ \\
& $F=\xi$ & $\rho_{s}>\lambda-$ \\
$0 \leq w<\frac{1}{9}$ & $\xi<F<\frac{1}{\kappa}$ & $\rho_{s}>\frac{1-\kappa F}{3 \kappa+3 \kappa w}$ \\
& $0<F<\frac{1+3 w}{2 \kappa-6 \kappa w}$ & $\frac{1-\kappa F}{3 \kappa+3 \kappa w}<\rho_{s}<\lambda_{+}$ \\
& $F=\frac{1+3 w}{2 \kappa-6 \kappa w}$ & $\frac{1-\kappa F}{3 \kappa+3 \kappa w}<\rho_{s}<\zeta$ \\
& $\frac{1+3 w}{2 \kappa-6 \kappa w}<F<\frac{1}{\kappa}$ & $\frac{1-\kappa F}{3 \kappa+3 \kappa w}<\rho_{s}<\lambda_{-}$ \\
& $\frac{1+3 w}{2 \kappa-6 \kappa w}<F<\frac{1}{\kappa}$ & $\rho_{s}>\lambda_{+}$ \\
$w \geq \frac{1}{9}$ & $0<F<\frac{1}{\kappa}$ & $\frac{1-\kappa F}{3 \kappa+3 \kappa w}<\rho_{s}<\lambda_{+}$ \\
\hline
\end{tabular}

of $\rho_{s}$ from homogeneous and inhomogeneous scalar perturbations, which indicates that the ES solution is unstable.

(ii) $F=\frac{1+3 w}{2 \kappa-6 \kappa w}$ and $\frac{1-\kappa F}{3 \kappa+3 \kappa w}<\rho_{s}<\zeta$. The inhomogeneous perturbations require $\rho_{s}$ to satisfy Eq. (54) when $k^{2}=8$. Since $\varpi>\zeta$, there is no stable ES solution in this case too.

(iii) $\frac{1+3 w}{2 \kappa-6 \kappa w}<F<\frac{1}{\kappa}$ and $\frac{1-\kappa F}{3 \kappa+3 \kappa w}<\rho_{s}<\lambda_{-}$. When $k^{2}=8, \rho_{s}$ is also required to satisfy Eq. (54). We find that $\varpi>\lambda_{-}$. Thus, the ES solution is unstable.

(iv) $\frac{1+3 w}{2 \kappa-6 \kappa w}<F<\frac{1}{\kappa}$ and $\rho_{s}>\lambda_{+}$. In this case, since the analytical results for the stability regions under inhomogeneous scalar perturbations are very complicated, we do not show them here and but resort to a numerical discussion. We find that when $n=2$ the smallest stability regions is obtained. With the increase of the value of $n$, the stability regions become larger and larger, which can be seen from Fig. 1. In this figure, $n$ is taken to be $n=0,2,3,4,5,6$, respectively, where $n=0$ corresponds to the case of homogeneous scalar perturbations.

$$
\begin{aligned}
\varpi= & \frac{7+[19+\kappa F(2-26 w)-27 w] \kappa F+5 w}{2 \kappa(1+w)[5+15 w+2 \kappa F(7+3 w)]} \\
& +\frac{1}{2} \sqrt{\frac{(1+2 \kappa F)^{2}\left[49+3 \kappa F(50+19 \kappa F)+70 w-2 \kappa F(34+57 \kappa F) w+(5+11 \kappa F)^{2} w^{2}\right]}{\kappa^{2}(1+w)^{2}[5+15 w+2 \kappa F(7+3 w)]^{2}}}
\end{aligned}
$$

where $k^{2}=8$ is taken. Since $\varpi>\lambda_{+}$for $0<w<\frac{1}{9}$ and $0<F<\frac{1+3 w}{2 \kappa-6 \kappa w}$, there is no overlap for the allowed regions
When $n \rightarrow \infty, b_{11}$ reduces to $b_{11} \simeq w k^{2}$. The stable ES solution requires 

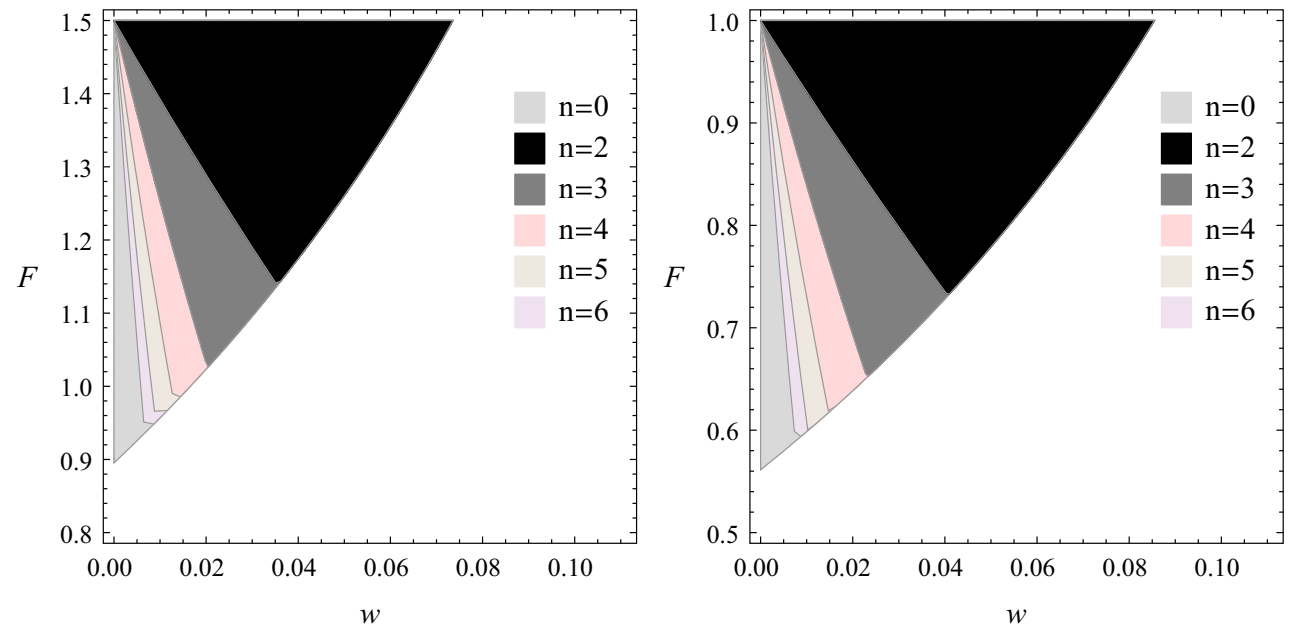

Fig. 1 The stability regions in the $F-w$ plane under homogeneous and inhomogeneous perturbations with $n$ taken to be $n=0,2,3,4,5,6 . n=0$ corresponds to the results from homogeneous perturbations. The left panel is plotted with $\rho_{s}=15$ and $\kappa=\frac{2}{3}$, while the right one for $\rho_{s}=15$ and $\kappa=1$

$$
\begin{aligned}
& M \simeq\left(w+\frac{1-\frac{\kappa}{a_{0}^{2}}}{1-3 \frac{\kappa}{a_{0}^{2}}}\right) k^{2}>0, \\
& M^{2}-N \simeq \frac{4 w\left(1-\frac{\kappa}{a_{0}^{2}}\right)}{1-3 \frac{\kappa}{a_{0}^{2}}} k^{4}>0 .
\end{aligned}
$$

The above two equations give

$-\frac{F}{1+w}<\rho_{s}<\frac{1-\kappa F}{3 \kappa+3 \kappa w}, \quad \rho_{s}>\frac{1+\kappa F}{\kappa+\kappa w}$

where $0<w<\frac{1}{9}$ and $\frac{1+3 w}{2 \kappa-6 \kappa w}<F<\frac{1}{\kappa}$ are considered together. Since $\frac{1+\kappa F}{\kappa+\kappa w}<\lambda_{+}$is always satisfied, the stability regions are larger than what are obtained under homogeneous scalar perturbations. Thus, in this case the ES solution is stable under both homogeneous and inhomogeneous scalar perturbations and the stability regions are given by taking $n=2$.

When $w \geq \frac{1}{9}$, we find that $M>0$ and $M^{2}-N>0$ can not be satisfied simultaneously, which shows that the stable ES solution does not exist.

\section{Conclusion}

In this paper, we have analyzed the stability of an ES universe under both scalar and tensor perturbations in gravity theory with a coupling between the kinetic term of the scalar field and the Einstein tensor. Homogeneous and inhomogeneous perturbations are considered together and inhomogeneous perturbations will compress the allowed regions of model parameters significantly. We find that the stable ES solution exists only in the spatially closed universe $(K=1)$ and it requires the coupling constant $\kappa>0$ to be positive. In addition, the equation of state of the perfect fluid is required to satisfy $0<w<\frac{1}{9}$, which indicates that if this perfect fluid is pressureless matter or radiation the stable ES solution does not exist, although it does under homogeneous perturbations. Thus, in the non-minimally kinetic coupled gravity with the perfect fluid satisfying $0<w<\frac{1}{9}$ the stable ES solution can exist under both scalar and tensor perturbations and the emergent mechanism can be used to avoid the big bang singularity.

When $F=0$, our results reduce to those obtained in [67] where the special condition $\dot{\phi}=0$ was considered. In this special case our analyses show that inhomogeneous scalar perturbations will break the stability of an ES solution although the solution is stable under tensor and homogeneous scalar ones. Therefore, the big-bang singularity problem cannot be solved successfully if $\dot{\phi}=0$ is taken.

Finally, a few comments are now in order for the emergent scenario proposed for avoiding the big-bang singularity. The emergent scenario assumes the existence of a stable Einstein static state and its past eternity. But usually such a state only exists under certain conditions. So, there is a question as to how this particular state comes into being in the first place, and in this regard, let us note that one possibility might be the creation of this state from "nothing" through quantum tunneling[106,107]. Another issue is that even if this state is stable classically, one still needs to address the question as to whether it is stable quantum mechanically, possibly by calculating the characteristic decay time in a quantum theory of cosmology when this state was formed. 
Acknowledgements This work was supported by the National Natural Science Foundation of China under Grants Nos. 11775077, 11435006, 11690034 , and 11375092.

Open Access This article is distributed under the terms of the Creative Commons Attribution 4.0 International License (http://creativecomm ons.org/licenses/by/4.0/), which permits unrestricted use, distribution, and reproduction in any medium, provided you give appropriate credit to the original author(s) and the source, provide a link to the Creative Commons license, and indicate if changes were made.

Funded by $\mathrm{SCOAP}^{3}$.

\section{References}

1. A.A. Starobinsky, Phys. Lett. B 91, 99 (1980)

2. A.H. Guth, Phys. Rev. D 23, 347 (1981)

3. A.D. Linde, Phys. Lett. B 108, 389 (1982)

4. M. Gasperini, G. Veneziano, Phys. Rep. 373, 1 (2003)

5. J.E. Lidsey, D. Wands, E.J. Copeland, Phys. Rep. 337, 343 (2000)

6. J. Khoury, B.A. Ovrut, P.J. Steinhardt, N. Turok, Phys. Rev. D 64, $123522(2001)$

7. P.J. Steinhardt, N. Turok, Science 296, 1436 (2002)

8. P.J. Steinhardt, N. Turok, Phys. Rev. D 65, 126003 (2002)

9. J. Khoury, P.J. Steinhardt, N. Turok, Phys. Rev. Lett. 92, 031302 (2004)

10. G.F.R. Ellis, R. Maartens, Class. Quantum Gravity 21, 223 (2004)

11. G.F.R. Ellis, J. Murugan, C.G. Tsagas, Class. Quantum Gravity 21, 233 (2004)

12. J.D. Barrow, G.F.R. Ellis, R. Maartens, C.G. Tsagas, Class. Quantum Gravity 20, L155 (2003)

13. A.S. Eddington, Mon. Not. R. Astron. Soc. 90, 668 (1930)

14. G.W. Gibbons, Nucl. Phys. B 292, 784 (1987)

15. G.W. Gibbons, Nucl. Phys. B 310, 636 (1988)

16. L.F. Abbott, Nucl. Phys. B 185, 233 (1981)

17. T. Futamase, K.I. Maeda, Phys. Rev. D 39, 399 (1989)

18. V. Sahni, S. Habib, Phys. Rev. Lett. 81, 1766 (1998)

19. J.P. Uzan, Phys. Rev. D 59, 123510 (1999)

20. F. Perrotta, C. Baccigalupi, S. Matarrese, Phys. Rev. D 61, 023507 (1999)

21. P.G. Bergmann, Int. J. Theor. Phys. 1, 25 (1968)

22. K. Nordtvedt, Astrophys. J. 161, 1059 (1970)

23. R. Wagoner, Phys. Rev. D 1, 3209 (1970)

24. T.P. Sotiriou, V. Faraoni, Rev. Mod. Phys. 82, 451 (2010)

25. A. De Felice, S. Tsujikawa, Living Rev. Relat. 13, 3 (2010)

26. C. Brans, R.H. Dicke, Phys. Rev. 124, 925 (1961)

27. R.H. Dicke, Phys. Rev. 125, 2163 (1962)

28. Planck Collaboration: P. A. R. Ade, N. Aghanim, M. Arnaud, et al., Astron. Astrophys. 594, A20 (2016)

29. J.D. Barrow, A.C. Ottewill, J. Phys. A 16, 2757 (1983)

30. C.G. Bohmer, L. Hollenstein, F.S.N. Lobo, Phys. Rev. D 76, 084005 (2007)

31. R. Goswami, N. Goheer, P.K.S. Dunsby, Phys. Rev. D 78, 044011 (2008)

32. N. Goheer, R. Goswami, P.K.S. Dunsby, Class. Quantum Gravity 26, 105003 (2009)

33. S. del Campo, R. Herrera, P. Labrana, J. Cosmol. Astropart. Phys. 07, 006 (2009)

34. S.S. Seahra, C.G. Bohmer, Phys. Rev. D 79, 064009 (2009)

35. H. Miao, P. Wu, H. Yu, Class. Quantum Gravity 33, 215011 (2016)

36. H. Huang, P. Wu, H. Yu, Phys. Rev. D 89, 103521 (2014)

37. S. Campo, R. Herrera, P. Labraña, J. Cosmol. Astropart. Phys. 11, 030 (2007)

38. S. Campo, R. Herrera, P. Labraña, J. Cosmol. Astropart. Phys. 07, 006 (2009)
39. S. Li, H. Wei, Phys. Rev. D 96, 023531 (2017)

40. P. Wu, H. Yu, Phys. Lett. B 703, 223 (2011)

41. J.T. Li, C.C. Lee, C.Q. Geng, Eur. Phys. J. C 73, 2315 (2013)

42. D.J. Mulryne, R. Tavakol, J.E. Lidsey, G.F.R. Ellis, Phys. Rev. D 71, $123512(2005)$

43. J.E. Lidsey, D.J. Mulryne, N.J. Nunes, R. Tavakol, Phys. Rev. D 70, 063521 (2004)

44. L. Parisi, M. Bruni, R. Maartens, K. Vandersloot, Class. Quantum Gravity 24, 6243 (2007)

45. R. Canonico, L. Parisi, Phys. Rev. D 82, 064005 (2010)

46. P. Wu, S. Zhang, H. Yu, J. Cosmol. Astropart. Phys. 05, 007 (2009)

47. S. Bag, V. Sahni, Y. Shtanov, S. Unnikrishnan, J. Cosmol. Astropart. Phys. 07, 034 (2014)

48. K. Zhang, P. Wu, H. Yu, L. Luo, Phys. Lett. B 758, 37 (2016)

49. K. Zhang, P. Wu, H. Yu, Phys. Lett. B 690, 229 (2010)

50. K. Zhang, P. Wu, H. Yu, Phys. Rev. D 85, 043521 (2012)

51. J.E. Lidsey, D.J. Mulryne, Phys. Rev. D 73, 083508 (2006)

52. A. Gruppuso, E. Roessl, M. Shaposhnikov, J. High Energy Phys. 08, 011 (2004)

53. L.A. Gergely, R. Maartens, Class. Quantum Gravity 19, 213 (2002)

54. K. Atazadeh, Y. Heydarzade, F. Darabi, Phys. Lett. B 732, 223 (2014)

55. K. Zhang, P. Wu, H. Yu, J. Cosmol. Astropart. Phys. 01, 048 (2014)

56. Y. Heydarzade, F. Darabi, K. Atazadeh, Astrophys. Space. Sci. 361, 250 (2016)

57. Y. Heydarzade, F. Darabi, J. Cosmol. Astropart. Phys. 04, 028 (2015)

58. H. Huang, P. Wu, H. Yu, Phys. Rev. D 91, 023507 (2015)

59. C.G. Bohmer, F.S.N. Lobo, Phys. Rev. D 79, 067504 (2009)

60. Q. Huang, P. Wu, H. Yu, Phys. Rev. D 91, 103502 (2015)

61. C.G. Bohmer, Class. Quantum Gravity 21, 1119 (2004)

62. K. Atazadeh, J. Cosmol. Astropart. Phys. 06, 020 (2014)

63. P. Wu, H. Yu, Phys. Rev. D 81, 103522 (2010)

64. C.G. Bohmer, F.S.N. Lobo, Eur. Phys. J. C 70, 1111 (2010)

65. M. Khodadi, Y. Heydarzade, F. Darabi, E.N. Saridakis, Phys. Rev. D 93, 124019 (2016)

66. C.G. Bohmer, N. Tamanini, M. Wright, Phys. Rev. D 92, 124067 (2015)

67. K. Atazadeh, F. Darabi, Phys. Lett. B 744, 363 (2015)

68. C.G. Bohmer, F.S.N. Lobo, N. Tamanini, Phys. Rev. D 88, 104019 (2013)

69. A.N. Tawfik, A.M. Diab, E.A.E. Dahab, T. Harko, Phys. Rev. D 93, 063526 (2016)

70. A.N. Tawfik, A.M. Diab, E.A.E. Dahab, T. Harko, arXiv: 1608.06532

71. M. Khodadi, Y. Heydarzade, K. Nozari, F. Darabi, Eur. Phys. J. C 75, $590(2015)$

72. S. Carneiro, R. Tavakol, Phys. Rev. D 80, 043528 (2009)

73. A. Odrzywolek, Phys. Rev. D 80, 103515 (2009)

74. T. Clifton, J.D. Barrow, Phys. Rev. D 72, 123003 (2005)

75. A. Vilenkin, Phys. Rev. D 88, 043516 (2013)

76. A. Aguirre, J. Kehayias, Phys. Rev. D 88, 103504 (2013)

77. A.T. Mithani, A. Vilenkin, J. Cosmol. Astropart. Phys. 01, 028 (2012)

78. Y. Cai, Y. Wan, X. Zhang, Phys. Lett. B 731, 217 (2014)

79. Y. Cai, M. Li, X. Zhang, Phys. Lett. B 718, 248 (2012)

80. M. Mousavi, F. Darabi, Nucl. Phys. B 919, 523 (2017)

81. H. Shabani, A.H. Ziaie, Eur. Phys. J. C 77, 31 (2017)

82. F. Darabi, K. Atazadeh, arXiv: 1704.03040

83. L. Amendola, Phys. Lett. B 301, 175 (1993)

84. S. Capozziello, G. Lambiase, Gen. Relativ. Gravit. 31, 1005 (1999)

85. S. Capozziello, G. Lambiase, H.J. Schmidt, Ann. Phys. 9, 39 (2000) 
86. L.N. Granda, JCAP 04, 016 (2011)

87. N. Yang, Q. Fei, Q. Gao, Y. Gong, Class. Quantum Gravity 33, 205001 (2016)

88. Y. Huang, Q. Gao, Y. Gong, Eur. Phys. J. C 75, 143 (2015)

89. C. Germani, A. Kehagias, JCAP 05, 019 (2010)

90. C. Germani, Y. Watanabe, JCAP 07, 031 (2011)

91. S. Tsujikawa, Phys. Rev. D 85, 083518 (2012)

92. H.M. Sadjadi, P. Goodarzi, Phys. Lett. B 732, 278 (2014)

93. F. Darabi, A. Parsiya, Class. Quantum Gravity 32, 155005 (2015)

94. S.V. Sushkov, Phys. Rev. D 80, 103505 (2009)

95. C. Gao, JCAP 06, 023 (2010)

96. A. Ghalee, Phys. Rev. D 88, 083528 (2013)

97. L.N. Granda, JCAP 07, 006 (2010)
98. L.N. Granda, W. Cardona, JCAP 07, 021 (2010)

99. L.N. Granda, Class. Quantum Gravity 28, 025006 (2011)

100. A.A. Starobinsky, S.V. Sushkov, M.S. Volkov, JCAP 1606, 007 (2016)

101. C. Germani, A. Kehagias, Phys. Rev. Lett. 105, 011302 (2010)

102. S.V. Sushkov, Phys. Rev. D 85, 123520 (2012)

103. E.N. Saridakis, S.V. Sushkov, Phys. Rev. D 81, 083510 (2010)

104. J.M. Bardeen, Phys. Rev. D 22, 1882 (1980)

105. E.R. Harrison, Rev. Mod. Phys. 39, 862 (1967)

106. J.B. Hartle, S.W. Hawking, Phys. Rev. D 28, 2960 (1983)

107. A. Vilenkin, Phys. Rev. D 30, 509 (1984) 